OPEN ACCESS

Edited by:

Sara Calderoni,

Fondazione Stella Maris (IRCCS), Italy

Reviewed by:

Margaret Lang Bauman, Boston University, United States

Enzo Grossi,

Villa Santa Maria Scs, Italy

*Correspondence:

María José Penzol

mj.penzol@gmail.com

Specialty section:

This article was submitted to Child and Adolescent Psychiatry,

a section of the journal

Frontiers in Psychiatry

Received: 27 September 2018 Accepted: 11 March 2019

Published: 10 April 2019

Citation:

Penzol MJ, Salazar de Pablo G Llorente C, Moreno C, Hernández $P$, Dorado ML and Parellada M (2019) Functional Gastrointestinal Disease in

Autism Spectrum Disorder: A

Retrospective Descriptive Study in a Clinical Sample.

Front. Psychiatry 10:179.

doi: 10.3389/fpsyt.2019.00179

\section{Functional Gastrointestinal Disease in Autism Spectrum Disorder: A Retrospective Descriptive Study in a Clinical Sample}

\author{
María José Penzol ${ }^{1,2 \star}$, Gonzalo Salazar de Pablo ${ }^{1}$, Cloe Llorente ${ }^{1}$, Carmen Moreno ${ }^{1}$, \\ Patricia Hernández ${ }^{1}$, Maria Luisa Dorado ${ }^{1}$ and Mara Parellada ${ }^{1}$ \\ ${ }^{1}$ Child and Adolescent Psychiatry Department, Hospital General Universitario Gregorio Marañón, School of Medicine, \\ Universidad Complutense, liSGM, CIBERSAM, Madrid, Spain, ${ }^{2}$ Molecular Medicine Ph.D Program, University of Santiago de \\ Compostela, Santiago de Compostela, Spain
}

Introduction: Autism spectrum disorder (ASD) is a heterogeneous group of neurodevelopmental disorders with complex multifactorial etiologies. Medical comorbidities are common in ASD and include functional gastrointestinal disorders (fGID), which are reported in $30-70 \%$ of patients. In this research study, we aimed to systematically assess the prevalence of gastrointestinal problems in ASD and describe their clinical correlates.

Methods: In this retrospective study, we reviewed the medical records of all patients admitted to the Comprehensive Medical Program for ASD (AMITEA) at Gregorio Marañón University General Hospital from January 2012 to December 2015. All patients fulfilled the clinical criteria for ASD (DSM-IV-TR). In addition to fGID, epidemiological and clinical variables were collected at intake. Clinical and demographic features were compared among subjects with and without comorbid gastrointestinal problems.

Results: The analyses included all patients with documented information about presence/absence of fGID ( $n=845 ; 95 \%$ of patients). Ages ranged from 1 to 53 years (mean $=10.52 ; \mathrm{SD}=8.92 ; 80.4 \%$ males). At least one fGID was present in $30.5 \%$ of patients, constipation being the most prevalent (47.4\% of fGID patients); fGID were significantly associated with intellectual disability (ID) $(p=0.017)$, sleep disorders $(p=0.012)$, and prescription of psychopharmacological treatment $(p=0.019)$.

Conclusions: Almost one-third of ASD patients in our sample had at least one fGID. The presence of fGID was associated with ID, sleep problems and with behavioral problems (as measured by the prescription of psychotropic drugs). This subsample of ASD patients with fGID deserves particular attention in future research projects, focusing on specific phenotypic characteristics and overlapping biological markers that may underlie both pathologies.

Keywords: functional gastrointestinal disease, autism spectrum disorder, clinical comorbidity, prevalence, retrospective study 


\section{INTRODUCTION}

Autism spectrum disorder (ASD) is a pervasive neurodevelopmental disorder characterized by impairments in social communication and restricted, repetitive patterns of behavior, interests, or activities (1). The neurobiological basis of ASD seems incontrovertible (2) even though the neurobiological mechanisms that result in the clinical phenotype remain to be fully elucidated. These include genetic factors, neuropathology, neurostructure, and brain networks (2). According to previous studies, more than $70 \%$ of individuals with ASD have other concurrent medical, developmental, or psychiatric conditions, which are frequently multiple (3-6).

It is widely reported that children with ASD are more likely to experience unmet medical needs compared with typically developing children (7). According to data from a US national survey, among children and adolescents with special health care needs, those with ASD are more likely to require specific health care services. This also holds true when compared with children who have special health care needs and behavioral, emotional, and developmental problems other than autism (8).

After years of debate about the presence of gastrointestinal (GI) symptoms in autism, there is currently a consensus to describe GI symptoms as a common comorbidity in patients with ASD even though the underlying mechanisms are largely unknown (9). A review published in Pediatrics reported that prevalence rates vary widely among studies and range from 9 to $91 \%$ in different samples, with great differences between retrospective and prospective studies $(10,11)$.

The GI problems, most commonly reported in autism, are chronic constipation, abdominal pain with or without diarrhea, and encopresis as a consequence of constipation (12). Furthermore, greater autism symptom severity is associated with increased odds of having GI problems $(11,13)$. Gastrointestinal symptoms are more likely to be reported by mothers in children with autism early in infancy than in children with typical development or developmental delay (14). Diagnosis of GI problems can be challenging in children with ASD because of their communication difficulties and complex behaviors (15), and therefore may be delayed (16), and problems frequently go undetected. GI symptoms in ASD may contribute to behavioral impairment, complicating clinical management. Gastrointestinal problems have been found to be associated with other behavioral and anxiety problems $(17,18)$.

Based on Roma Foundation working team, functional gastrointestinal disorders (fGIDs) are described as gut-brain interaction disorders, defined as a resulting on combination of symptoms affecting motility, hypersensitivity, immunity, and other alterations in mucose, causing an illness experience in patient's body; which are not caused by a anatomic or motility disorder (19).

A high prevalence of sleeping problems has been found in children with ASD (20-22), with longer sleep latencies and more difficulty going to bed and falling asleep (23). Co-occurrence of GI and sleep disturbances have been widely described in ASD, suggesting potential common pathophysiological pathways (24).
There are common pathophysiological mechanisms that account for both autism and epilepsy (25). ASD and epilepsy cooccur in approximately $30 \%$ of individuals with either condition (26). An epidemiological study detected an association between GID and epilepsy, identifying an increased risk for seizures co-occurring with GI symptoms in ASD patients (27).

It has been suggested that high medical comorbidity in ASD subjects may be due to common etiopathological factors or shared intermediate mechanisms, such as inflammation, immunity, redox status, etc. (16). However, an association of medical comorbidities with fGID in a sample of ASD subjects representative of the ASD general population has not yet been sufficiently explored.

Against this background, the purpose of this work was to explore fGID and associated conditions in a large sample of patients consecutively seen in a medical program for ASD subjects over a representative period of time.

In this study, we specifically evaluated (1) the prevalence of fGID in a large sample of individuals with ASD quasirepresentative of the population of ASD patients in the Community of Madrid and (2) the sociodemographic and clinical characteristics and comorbidities in the subgroup of ASD patients with fGID.

\section{MATERIALS AND METHODS}

We conducted a retrospective study reviewing clinical records of all patients admitted to the Comprehensive Medical Program for ASD (AMITEA) (28) at Gregorio Marañón University General Hospital during the first four full years after implementing an electronic medical records system: from January 1, 2012 to December 31, 2015. The AMITEA program was founded in 2009 as a specialty care service for ASD patients with specific objectives: (1) to perform routine and symptom- or sign-driven medical assessments, (2) to facilitate access to the appropriate medical care using an individualized case management approach, (3) to centralize all possible medical and nursing procedures, and (4) to assess and treat comorbid psychiatric symptomatology (28). The only criteria for admission to the program are: (1) diagnosis of an ASD (all ages); (2) referral by a physician in the public sector; and (3) residing in the Community of Madrid (thus serving a population of 6,000,000). AMITEA is located in a tertiary hospital with all adult and pediatric specialties and more than 2,000 inpatient beds. Spain has a national health system that covers around $95 \%$ of the population. Given that the AMITEA program is open to all residents in the Community of Madrid Community with an ASD diagnosis (the only program of its kind) and admission is not restricted to individuals with health problems, the characteristics of program participants may be considered representative of the ASD general population in Madrid.

All participants fulfilled DSM-IV-TR criteria for ASD. Diagnoses were made by Child and Adolescent Psychiatrists, after a full psychiatric and developmental history, previous reports' review and observation of the child/adolescent. The Autistic Diagnostic Interview-Revised (ADI-R) and Autism Diagnostic 
Observation Schedule-Generic ADOS-G were used to support the diagnosis when clinicians deemed it necessary. When needed, ADI-R clinically trained and ADOS research trained child psychiatrists or psychologists did conduct these evaluations. All data was introduced in the electronic medical history of the Hospital at the time of intake, and electronically signed. In order to organize the information from the hospital medical history and report the data herein presented, we built a database in which patients' data were de-identified.

All data were collected as part of routine care, according to a protocol completed for every new participant registered in AMITEA. Sociodemographic and clinical variables were collected systematically by attending physicians (Child and Adolescent Psychiatrists). Data collected included age, sex, ASD diagnosis, type of ASD, and presence of fGID (gastrointestinal reflux, aerophagia, functional diarrhea, functional constipation, functional abdominal pain, cyclic vomiting). Specifics of the fGID, if present, were manually extracted from the electronic medical record and entered verbatim in the study database. Within variables deemed interesting with respect to gastrointestinal comorbidity, we only analyzed data that was included in the initial intake clinical history template. In this way, the data we report herein were available for all patients. GI information was categorized according to the domains in the ROME-III-R questionnaire for fGID (Appendix. Rome Foundation, 2006). Other comorbidities such as epilepsy, estimated intellectual disability (ID), and sleep disorders were also compiled. Behavioral and emotional problems were recorded as part of the psychiatric history, with no standard questionnaire; therefore, prescription of psychotropic treatment was gathered as a proxy for the behavioral problems (with clinical impact). Estimated ID was recorded as a dichotomous (yes/no) clinical variable after reviewing all the information in the medical record including psychometric tests, adaptive functioning questionnaires, and school placement and performance. The Institutional Review Board (IRB) of Gregorio Marañón University General Hospital approved the study.

\section{ANALYSES}

We used descriptive statistics to characterize the study population including demographic variables and clinical characteristics. These were compared between patients groups with and without comorbid GI problems. A logistic regression model was then used to estimate odds ratios (ORs) and explore how factors differentially distributed between patients with and without fGID in the bivariate analyses contributed to the association with fGID. Therefore, we included age, sex, intellectual disability and sleep disorder as covariates in the multivariate analysis. Statistical analyses were performed with SPSS 21 for Windows software (IBM).

\section{RESULTS}

Our analyses included all patients with available information about presence/absence of fGID $(n=845 ; 95.6 \%)$ of a total sample of 879 persons admitted in the AMITEA program during 4 years; therefore, 34 patients were excluded from analysis since data of interest were not fully completed in clinical records. Ages ranged from 1 to 53 years, with a mean age of 10.52 (SD 8.92), and $19.6 \%$ were female. Children under 5 years of age comprised $25.2 \%$ of the sample $(n=213), 43 \%(n=363)$ were ages $5-12$, $15.9 \%(n=134)$ were adolescents ages $13-17$, and $16 \%(n=$ 135) were 18 or older. Four children aged 21-23 months were included in the program (following criteria as in the moderateto-severe concern in Toddler module of the ADOS). Per DSM-IV criteria, all patients had a diagnosis of Pervasive Developmental Disorder; most patients $(n=476 ; 56.3 \%)$ had a diagnosis of Autistic Disorder, 210 (24.9\%) had a diagnosis of Pervasive Developmental Disorder Not Otherwise Specified, 116 (13.7\%) Asperger's Disorder, 2 patients (0.2\%) had Rett's Disorder; and a small group $(n=41 ; 4.9 \%)$ did not had an specified subtype in the clinical record.

Thirty percent of subjects $(n=258,30.5 \%)$ had an fGID, constipation being the most prevalent, occurring in almost half ( $n=121 ; 47.6 \%)$ of the sub-sample with fGID; other symptoms identified were functional diarrhea ( $n=29 ; 11.4 \%)$, gastrointestinal reflux $(n=20 ; 7.9 \%)$, functional abdominal pain ( $n=16 ; 6.3 \%)$, aerophagia $(n=16 ; 6.3 \%)$, cyclic vomiting ( $n=12 ; 4.7 \%)$, and other fGID not specifically reported ( $n$ $=40 ; 15.7 \%)$. Eighty-four patients $(9.9 \%)$ of total sample were referred to a gastroenterologist for assessment, in this subgroup $75 \%$ presented fGID $\left(\chi^{2}=87.609, p<0.001\right)$.

With respect to the other comorbidities explored, $34.9 \%$ of the patients had a sleep disorder, 9.2\% epilepsy, and $44.3 \%$ intellectual disability. More than half of the patients in the sample (55\%) were prescribed psychopharmacological treatment. Psychotropic medication was more frequently used in patients over 12 years of age $\left(\chi^{2}=79.184 p=0.015\right)$, with a similar frequency in adolescents (79.9\%) and adults (79.5\%).

In contrast to the $30.5 \%$ of fGID in total sample, within the subsample of patients with ID, fGID was present in $34.8 \%$, while in patients without ID, fGID was present in 65.2\% ( $p=0.017)$.

With respect to the differential sociodemographic and clinical characteristics of patients with and without fGID (see Table 1). The percentage of patients with ID was greater in the group with fGID ( $p=0.017$ ), as was in the sleep disorder group $(p=0.012)$, and the group with prescription of psychotropic drugs $(p=0.019)$. Other clinical variables did not differ in both groups. No differences concerning age and gender were found between groups with and without fGID.

Most patients with sleep disorders (71.8\%) were on psychopharmacological treatment, while only $28.2 \%$ of patients with sleep disorders were receiving no psychotropic medication $\left(\chi^{2}=31.732, p<0.001\right)$.

Exploring eventual predictors of fGID, we examined the variables associated with functional Gastrointestinal Disorders (see Table 2), logistic regression analysis showed that intellectual disability was significantly associated with fGID (OR $=1.490$, 95\% CI: $1.081-2.055 ; p=0.015)$ and sleep disorder $(\mathrm{OR}=1.502$, 95\% CI:1.107-1.2036; $p=0.009$ ) were significantly associated with fGID. Result remained significant in the multivariate analysis when OR was adjusted for age, sex, and sleep problems; 
and also when OR for sleep problems when covariated by age, sex, and intellectual disability. Due to the high association between sleep disorder and psychopharmacological treatment, this last variable was not included in the regression.

\section{DISCUSSION}

In this sample of 845 patients with ASD seen in a specialty care program for ASD patients in a tertiary hospital, almost a third had an fGID, constipation being the most prevalent (47\%). The presence of fGID was associated with ID, sleep problems and with behavioral problems (as measured by the

TABLE 1 | Sociodemographic and clinical characteristics in the groups with and without fGID.

\begin{tabular}{|c|c|c|c|c|}
\hline & $\begin{array}{c}\text { fGID } \\
N=258\end{array}$ & $\begin{array}{l}\text { No fGID } \\
N=587\end{array}$ & $\mathrm{X}^{2}$ test & $p$-value \\
\hline Age groups & & & 0.043 & 0.998 \\
\hline Age $\leq 5$ (infants/toddlers) & $24.8 \%$ & $25.4 \%$ & & \\
\hline Age 5-12 (children) & $43.4 \%$ & $42.8 \%$ & & \\
\hline Age 13-18 (adolescents) & $15.9 \%$ & $15.8 \%$ & & \\
\hline Age $\geq 18$ (adults) & $15.9 \%$ & $16.0 \%$ & & \\
\hline Sex (female) & $20.9 \%$ & $19.1 \%$ & 0.389 & 0.573 \\
\hline ASD diagnostic category & & & 3.634 & 0.603 \\
\hline Autistic Disorder & $63 \%$ & $57.5 \%$ & & \\
\hline Rett's Disorder & $0 \%$ & $0.4 \%$ & & \\
\hline Asperger's Disorder & $12.2 \%$ & $15.4 \%$ & & \\
\hline PDD NOS & $24.8 \%$ & $26.7 \%$ & & \\
\hline Known medical etiology & $19.4 \%$ & $18.5 \%$ & 0.099 & 0.753 \\
\hline Epilepsy & $11.8 \%$ & $9.1 \%$ & 1.457 & 0.247 \\
\hline Intellectual disability & $51.2 \%$ & $41.3 \%$ & 5.946 & $0.017^{\star}$ \\
\hline Sleep disorder & $42.7 \%$ & $33.2 \%$ & 6.878 & $0.009^{*}$ \\
\hline Pharmacological treatment & $65.3 \%$ & $56.4 \%$ & 5.563 & $0.019^{*}$ \\
\hline
\end{tabular}

PDD-NOS, Pervasive Developmental Disorder-Not otherwise specified.

${ }^{*}$ Bold values indicate statistically significant difference $(p<0.05)$. higher percentage of children in the ASD group with fGID treated with psychotropic drugs), but it was intellectual disability and sleep problems that stood out as the most significant associations with fGID once other variables were considered. No differences were detected in the prevalence of fGID among age groups or ASD diagnosis category.

In this large ASD sample, relatively representative of ASD subjects in a general population of around 6,000,000, there emerges a subgroup of patients (around 25\% of the sample) characterized by GID and ID. This group of patients has concomitant associated health problems such as sleep and behavioral problems. Although parents with ID report fGID only a bit more frequently than parents of children without ID (34.8 vs. $30.5 \%, p=0.017)$, the percentage could well be higher, taking into account the difficulties that ASD+ID patients may have communicating symptoms, especially if those include subjective perceptions such as pain or discomfort.

Our finding of nearly one-third of ASD patients presenting with fGID adds to the previous literature. A recent large population-based study (17) showed that $7.5 \%$ of ASD participants had a gastrointestinal problem, the most common being constipation. One-third of ASD patients presenting with fGID in a tertiary hospital is a higher figure than the $11.7 \%$ found in a recent study that automatically searched a large sample of medical records from four general hospitals (29) for comorbidities. Our data is likely more accurate, as the presence of fGID is systematically recorded at the intake visit in a program specifically designed to meet the medical needs of patients with ASD (2) referred by general practitioners. The nature of this program, designed to improve access to specialist care via a case manager, and the nature of the healthcare system in Spain, which is publicly funded and available to all citizens, results in a case load of patients either seen for any medical (including psychiatric) comorbidity or simply registered in the program should they need any specialty.

TABLE 2 | Functional Gastrointestinal Disorder.

\begin{tabular}{|c|c|c|c|c|c|c|}
\hline & Unadjusted OR & $(95 \% \mathrm{Cl})$ & $p$-value & Adjusted OR ${ }^{a / b}$ & $(95 \% \mathrm{Cl})$ & $p$-value \\
\hline Age & 1.002 & $0.986-1.019$ & 0.801 & - & - & - \\
\hline Sex (male) & 0.891 & $0.619-1.082$ & 0.533 & - & - & - \\
\hline ASD diagnostic category & 1 & $0.457-1.142$ & 0.164 & - & - & - \\
\hline Autistic disorder & 0.722 & & 0.361 & - & - & - \\
\hline Asperger's Disorder & 0.848 & $0.595-1.208$ & & - & - & - \\
\hline PPD NOS & & & & - & - & - \\
\hline Known medical etiology & 0.942 & $0.649-1.367$ & 0.753 & - & - & - \\
\hline Epilepsy & 1.348 & $0.829-2.192$ & 0.229 & - & - & - \\
\hline Intellectual disability & 1.490 & $1.081-2.055$ & $0.015^{\star}$ & $1.402^{a}$ & $1.001-1.962$ & $0.049^{*}$ \\
\hline Sleep disorder & 1.502 & $1.107-2.036$ & $0.009^{\star}$ & $1.551^{\mathrm{b}}$ & $1.112-2.164$ & $0.010^{*}$ \\
\hline Pharmacological treatment & 1.455 & $1.065-1.988$ & $0.019^{\star}$ & - & - & - \\
\hline
\end{tabular}

${ }^{a}$ Adjusting for age, sex, and sleep disorder.

${ }^{b}$ Adjusting for age, sex, and intellectual disability.

PDD-NOS, Pervasive Developmental Disorder-Not otherwise specified.

${ }^{*}$ Bold values indicate statistically significant difference $(p<0.05)$. 
In our sample, functional gastrointestinal disorders were frequently associated with sleep disorders. According to the literature, GID and sleep problems are prevalent in pediatric samples without autism (around 45\%) (30). In samples with autism, sleep problems are more prevalent than in general population; two-thirds of children with ASD have chronic insomnia (21), which may cause daytime behavior, memory, and learning problems in patients, and significant stress in caretakers (20). Medical conditions more frequently overlapped in autism samples; some authors have even suggested that patients with GI and/or sleep problems may represent a differential subtype of ASD with potential common pathophysiological pathways $(22,24,27)$. GIDs have been described as a risk factor for sleep problems in children and adolescents with idiopathic autism (31).

Although the direction of the causal relationship between sleep problems and fGID is not known in many of the cases, early identification and treatment of GI disorders could improve sleep and help increase functionality and well-being in patients with ASD. In some cases, sleep problems clearly seem to arise from abdominal discomfort. As an example, a high percentage (40\%) of some monogenic forms of autism, such as PhelanMcDermid syndrome, present a specific gastrointestinal disorder, namely gastroesophageal reflux (32). This is frequently associated with sleep problems. Understanding the origin and associations of sleep problems in specific cases may help put them in the appropriate place and determine the specific treatment intervention (in this case, antacid treatment), preventing children taking ineffective and potentially damaging treatments (e.g., psychotropics for sleep disturbances).

GI problems have been described in the literature in association with behavioral problems in patients with $\operatorname{ASD}(6,22)$ and also with autism severity $(17,18,33)$. Although our data focused only on the use of psychopharmacological treatment, it could be considered an approximation of the presence of this problem in our sample.

Identifying clinical phenotypes in ASD via medical comorbidities may be a promising approach for improving our understanding of the pathophysiology of subgroups of patients and optimizing therapeutic interventions (34). Wasilewska and Klukowsky suggest comorbidity of GI disorders and $\mathrm{ASD}$ as a new endophenotype and propose treating it as an "overlap syndrome" through different mechanisms. These mechanisms include multilevel pathways in the gut-brain axis contributing to alterations in behavior and cognition (16).

There is considerable evidence that the gut-brain axis is involved in the etiology of autism. This is based on a number of studies focusing on permeability of the intestinal mucosa, abnormal gut development, leaky gut, and others, with different mechanisms involving different systemic processes and the intermediate mechanism, many of those pointing toward a systemic chronic pro-inflammatory status. Furthermore, for the last several years, there has been an escalating number of studies showing changes of gut microbiota in patients with ASD (35).

\section{LIMITATIONS}

We are aware that this study is subject to some limitations. Above all, it is a retrospective study, based on data recorded in medical records with only part of the clinical data potentially relevant to the topic of this study and systematically gathered. Since this is a transversal study, no data is available concerning how treatment of functional gastrointestinal symptoms could change patients' behavior. In addition, some clinical data potentially relevant for interpreting our results was not systematically recorded and could not therefore be analyzed.

Despite the above limitation, our study has important strengths. The major strength is the large sample size and its particularities, making it a sample quite representative of a population of children and adults with ASD.

In conclusion, a large sample of ASD patients from a tertiary hospital shows a subgroup of patients with fGID, half of them with intellectual disability and frequently with comorbid sleep problems and/or behavioral problems. This is a subgroup of patients with greater medical needs that need to be addressed, on top of their autistic core symptoms, in order to improve their well-being and consequently their adjustment.

\section{ETHICS STATEMENT}

This study was conducted following the recommendations of the IRB of our institution and the Spanish Agency of Medicines and Medical Devices (AEMPS). Waiver of individual informed consent was approved by the IRB.

\section{AUTHOR CONTRIBUTIONS}

MJP and MP were responsible for conception and design of the study. MJP, GS, CL, PH, CM, MD, and MP were responsible for the clinical interview with patients and recorded data from clinical records. MJP and GS conducted the database generation and drafted the article. MJP and MP performed statistical analysis and data interpretation. MJP, GS, CL, PH, CM, MD, and MP reviewed the manuscript, made substantial contributions to conception and data acquisition and interpretation, making modifications as appropriate to the work in progress. All authors agree with the final content of this work.

\section{ACKNOWLEDGMENTS}

Supported by the Spanish Ministry of Science, Innovation and Universities (PI14/02103), co-financed by ERDF Funds from the European Commission, A way of making Europe, CIBERSAM, Madrid Regional Government (B2017/BMD-3740 AGES-CM2), European Union Structural Funds and European Union Seventh Framework Program and H2020 Program (under the Innovative Medicines Initiative 2 Joint Undertaking (grant agreement No. 777394, Project AIMS-2-TRIALS); Fundación Familia Alonso, Fundación Alicia Koplowitz, and Fundación Mutua Madrileña. 


\section{REFERENCES}

1. American Psychiatric Association. Diagnostic and Statistical Manual of Mental Disorders, 5th Edition (DSM-5), Diagnostic and Statistical Manual of Mental Disorders 4th edition TR. American Psychiatric Association (2013).

2. Parellada M, Penzol MJ, Pina L, Moreno C, González-Vioque E, Zalsman G, et al. The neurobiology of autism spectrum disorders. Euro Psychiatry. (2014) 29:11-9. doi: 10.1016/j.eurpsy.2013.02.005

3. Simonoff E, Pickles A, Charman T, Chandler S, Loucas T, Baird G. Psychiatric disorders in children with autism spectrum disorders: prevalence, comorbidity, and associated factors in a populationderived sample. J Am Acad Child Adolesc Psychiatry. (2008) 47:921-9. doi: 10.1097/CHI.0b013e318179964f

4. Lai M-C, Lombardo MV, Baron-Cohen S. Autism. Lancet. (2014) 383:896910. doi: 10.1016/S0140-6736(13)61539-1

5. Levy SE, Giarelli E, Lee LC, Schieve LA, Kirby RS, Cunniff C, et al. Autism spectrum disorder and co-occurring developmental, psychiatric, and medical conditions among children in multiple populations of the United States. J Dev Behav Pediatr. (2010) 31:267-75. doi: 10.1097/DBP.0b013e3181d5d03b

6. Soke GN, Maenner MJ, Christensen D, Kurzius-Spencer M, Schieve LA. Prevalence of co-occurring medical and behavioral conditions/symptoms among 4- and 8-year-old children with autism spectrum disorder in selected areas of the United States in 2010. J Autism Dev Disord. (2018) 48:2663-76. doi: 10.1007/s10803-018-3521-1

7. Chiri G, Warfield ME. Unmet need and problems accessing core health care services for children with autism spectrum disorder. Mater Child Health J. (2012) 16:1081-91. doi: 10.1007/s10995-011-0833-6

8. Kogan MD, Strickland BB, Blumberg SJ, Singh GK, Perrin JM, van Dyck PC. A national profile of the health care experiences and family impact of autism spectrum disorder among children in the United States, 2005-2006. Pediatrics. (2008) 122:e1149-158. doi: 10.1542/peds.2008-1057

9. Mayer EA, Padua D, Tillisch K. (2014). Altered brain-gut axis in autism: comorbidity or causative mechanisms? BioEssays. 36:933-9. doi: 10.1002/bies.201400075

10. Buie T, Campbell DB, Fuchs GJ, Furuta GT, Levy J, Vandewater J, et al. Evaluation, diagnosis, and treatment of gastrointestinal disorders in individuals with asds: a consensus report. Pediatrics. (2010) 125(Suppl 1):S118. doi: 10.1542/peds.2009-1878C

11. Holingue C, Newill C, Lee LC, Pasricha PJ, Daniele Fallin M. Gastrointestinal symptoms in autism spectrum disorder: a review of the literature on ascertainment and prevalence. Autism Res. (2018) 11:24-36. doi: 10.1002/aur.1854

12. Rosenfeld CS. Microbiome disturbances and autism spectrum disorders. Drug Metab Dispos Biol Fate Chem. (2015) 43:1557-71. doi: $10.1124 / \mathrm{dmd} .115 .063826$

13. Wang LW, Tancredi DJ, Thomas DW. The prevalence of gastrointestinal problems in children across the United States with autism spectrum disorders from families with multiple affected members. J Develop Behav Pediatr. (2011) 32:351-60. doi: 10.1097/DBP.0b013e31821bd06a

14. Bresnahan M, Hornig M, Schultz AF, Gunnes N, Hirtz D, Lie KK, et al. Association of maternal report of infant and toddler gastrointestinal symptoms with autism: evidence from a prospective birth cohort. JAMA Psychiatry. (2015) 72:466-74. doi: 10.1001/jamapsychiatry.2014.3034

15. Kral TV, Eriksen WT, Souders MC, Pinto-Martin JA. Eating behaviors, diet quality, and gastrointestinal symptoms in children with autism spectrum disorders: a brief review. J Pediatr Nurs. (2013) 28:548-56. doi: 10.1016/j.pedn.2013.01.008

16. Wasilewska J, Klukowski M. Gastrointestinal symptoms and autism spectrum disorder: links and risks - a possible new overlap syndrome. Pediatric Health Med Ther. (2015) 6:153-66. doi: 10.2147/PHMT.S85717

17. Chaidez V, Hansen RL, Picciotto IH, et al. Gastrointestinal problems in children with autism, developmental delays or typical development. J Autism Dev Disord. (2014) 44:1117-27. doi: 10.1007/s10803-013-1973-x

18. Fulceri F, Morelli M, Santocchi E, Cena H, Del Bianco T, Narzisi A, et al. Gastrointestinal symptoms and behavioral problems in preschoolers with autism spectrum disorder. Dig Liver Dis. (2016) 48:248-54. doi: 10.1016/j.dld.2015.11.026

19. Drossman DA. Functional gastrointestinal disorders: history pathophysiology clinical features and rome IV. Gastroenterology. (2016). doi: 10.1053/j.gastro.2016.02.032
20. Kotagal S, Broomall E. Sleep in children with autism spectrum disorder Pediatr Neurol. (2012) 11:242-51. doi: 10.1016/j.pediatrneurol.2012.05.007

21. Souders MC, Zavodny S, Eriksen W, Sinko R, Connell J, Kernset C, et al. Sleep in children with autism spectrum disorder. Curr Psychiatry Rep. (2017) 19:34. doi: 10.1007/s11920-017-0782-x

22. Yang XL, Liang S, Zou MY, Sun $\mathrm{CH}$, Han PP, Jiang XT, et al. Are gastrointestinal and sleep problems associated with behavioral symptoms of autism spectrum disorder? Psychiatry Res. (2018) 259:229-35. doi: 10.1016/j.psychres.2017.10.040

23. Goldman SE, Alder ML, Burgess HJ, Corbett BA, Hundley R, Wofford D, et al. Characterizing sleep in adolescents and adults with autism spectrum disorders. J Autism Dev Disord. (2017) 47:1682-95. doi: 10.1007/s10803-017-3089-1

24. Neumeyer AM, Anixt J, Chan J, Perrin JM, Murray D, Coury DL, et al. Identifying associations among co-occurring medical conditions in children with autism spectrum disorders. Acad Pediatr. (2018). doi: 10.1016/j.acap.2018.06.014

25. Tuchman R, Rapin I. Epilepsy in autism. Lancet Neurol. (2002) 352-8. doi: 10.1016/S1474-4422(02)00160-6

26. Tuchman R, Alessandri M, Cuccaro M. Autism spectrum disorders and epilepsy: moving towards a comprehensive approach to treatment. Brain Dev. (2010) 31:719-30. doi: 10.1016/j.braindev.2010.05.007

27. Aldinger KA, Lane CJ, Veenstra-VanderWeele J, Levitt P. Patterns of risk for multiple co-occurring medical conditions replicate across distinct cohorts of children with autism spectrum disorder. Autism Res. (2015) 8:771-81. doi: 10.1002/aur.1492

28. Parellada M, Boada L, Moreno C, Llorente C, Romo J, Muela C, et al. Specialty Care programme for autism spectrum disorders in an urban population: a case-management model for health care delivery in an ASD population. Eur Psychiatry. (2013) 28:102-9. doi: 10.1016/j.eurpsy.2011.06.004

29. Kohane IS, McMurry A, Weber G, MacFadden D, Rappaport L, Kunkel L, et al. The co-morbidity burden of children and young adults with autism spectrum disorders. PLoS ONE. (2012) 7:e33224. doi: 10.1371/journal.pone.00 33224

30. Schurman JV, Friesen CA, Dai H, Danda CE, Hyman PE, Cocjin JT. (2012). Sleep problems and functional disability in children with functional gastrointestinal disorders: an examination of the potential mediating effects of physical and emotional symptoms. BMC Gastroenterol. (2012) 12:142. doi: 10.1186/1471-230X-12-142

31. McCue LM, Flick LH, Twyman KA, Xian H. Gastrointestinal dysfunctions as a risk factor for sleep disorders in children with idiopathic autism spectrum disorder: a retrospective cohort study. Autism. (2017) 21:1010-20. doi: $10.1177 / 1362361316667061$

32. Soorya L, Kolevzon A, Zweifach J, Lim T, Dobry Y, Schwartz L, et al. Prospective investigation of autism and genotype-phenotype correlations in 22q13 deletion syndrome and SHANK3 deficiency. Mol Autism. (2013) 4:18. doi: $10.1186 / 2040-2392-4-18$

33. Adams JB, Johansen LJ, Powell LD, Quig D, Rubin RA. Gastrointestinal flora and gastrointestinal status in children with autism-comparisons to typical children and correlation with autism severity. BMC Gastroenterol. (2011) 11:22. doi: 10.1186/1471-230X-11-22

34. Bauman ML. Medical comorbidities in autism: challenges to diagnosis and treatment. Neurotherapeutics. (2010) 7:320-7. doi: 10.1016/j.nurt.2010. 06.001

35. Tomova A, Husarova V, Lakatosova S, Bakos J, Vlkova B, Babinska $\mathrm{K}$, et al. Gastrointestinal microbiota in children with autism in Slovakia. Physiol Behav. (2015) 138:179-87. doi: 10.1016/j.physbeh.201 4.10 .033

Conflict of Interest Statement: The authors declare that the research was conducted in the absence of any commercial or financial relationships that could be construed as a potential conflict of interest.

Copyright (๑) 2019 Penzol, Salazar de Pablo, Llorente, Moreno, Hernández, Dorado and Parellada. This is an open-access article distributed under the terms of the Creative Commons Attribution License (CC BY). The use, distribution or reproduction in other forums is permitted, provided the original author(s) and the copyright owner(s) are credited and that the original publication in this journal is cited, in accordance with accepted academic practice. No use, distribution or reproduction is permitted which does not comply with these terms. 